\title{
Service-oriented Computation in Magnetic Fusion Research
}

\author{
David Schissel \\ General Atomics
}

\begin{abstract}
Fusion science seeks a new power source and is advanced by experiments on fusion devices located worldwide. Fundamental to increasing understanding of fusion is the comparison of theory and experiment; measurements from fusion devices are analyzed and compared with the output of simulations to test the validity of fusion models and to uncover new physical properties. Integrating simulations with experimental data (or with other simulations) is in many cases a labor-intensive task as different codes use different data storage formats. Moreover, the timely comparison of simulation with observations made during an experiment requires rapid turnaround both of analysis codes and simulation runs. Many simulations require extensive input and output processing, further increasing the amount of work necessary to achieve viable scientific results. Workers with the National Fusion Collaboratory are developing, deploying, and evaluating new technologies that facilitate analysis of experimental data and comparison with the results with those of simulations. Complex physics codes are made available on the National Fusion Grid (FusionGrid) as comprehensive computational services. Using the Globus Toolkit", a service-based approach was developed and subsequently combined with the TRANSP transport code to the benefit of fusion scientists. Output from both simulation and experimental codes are stored in MDSplus, the de facto standard for secure data storage of fusion data. Access control for the resources of FusionGrid is greatly simplified_for both users and administrators_through unified authentication and authorization using X.509, a grid-wide certificate management system, and a grid-wide authorization system. Web-based solutions such as the recently developed Elfresco reflectometry code further simplify the process by making simulations available to scientists and providing an alternative to traditional distribution of code. Future work includes the development of parallelized modules to speed up long-running codes along with the extension of MDSplus. These
\end{abstract}


improvements will help accommodate the continuous data streams that will be found in future fusion devices such as ITER. This paper will present a discussion on specific solutions, examine deployment areas that present a challenge, and highlight areas where further work is required. 


\section{Q\&A - David Schissel}

\section{Questioner: David Walker}

Could you comment on the similarities and dissimilarities between how the fusion community is using the grid and how the HEP community is using it; e.g. for analyzing LHC data?

\section{David Schissel}

The HEP/LHC community is using grid technology to move huge quantities of data to Tier sites for analysis that occurs after the experiment. For the experimental fusion community the quantity of data is not nearly as large. Thus, our usage of the grid is for more interactive work with data as well as providing computational services. Recent discussions between the fusion and HEP communities have identified areas of commonality between what FusionGrid has accomplished and what is needed by HEP to monitor (not analyze), in real-time ongoing experiments. The communities are examining ways to work together for their mutual benefit. It should be noted that both communities are using the grid for human communication with Access Grid and VRVS/EVO technology.

\section{Questioner: Brian Smith}

What is the current reliability levels (of network, computation, experimental (fusion systems)?

\section{David Schissel}

Network connectivity for fusion research in the United States is provided by ESnet with a reliability (this is from my memory) of somewhere between three and five 9's. The complex Tokamak systems that include power supplies, heating systems, diagnostics and computers are less reliable than ESnet but this is due to the lack of redundant systems whose cost would be prohibitive.

\section{Questioner: William Gropp}

You said a goal was to create a "standard toolset." What does standard mean here? Follow-up: How did you achieve your community standards?

\section{David Schissel}

Within the experimental fusion community there is a history of sharing computer codes rather than each individual institution solving the same problem. Most likely the best example of this is the MDSplus data acquisition and management system. My statements regarding a standard toolset were made within this context and were not meant to imply that a standards body would be created for a formal declaration. Our community's standards are created informally but good communication between the major organizations 
is critical. The history of communication and sharing within our community makes this a rather straightforward exercise.

\section{Questioner: Ronald Boisvert}

You explained that securitylauthentication issues remain a challenge to scaling your system to larger number of users. Are there other middleware type systems that are causing similar problems for you? What are the critical systems and services that need improvements for builders of grids systems to use?

\section{David Schissel}

Security issues are by far on the top of our list. Another item we are still working towards is a solution for federated web portals that are relatively easy for scientific users to interact with and to add services to. The other issue that has been a problem for us is network multicast. Although ESnet supports this we find that many local LANs do not.

\section{Comment: Mary Thomas}

It is difficult to get Fusion Security people to open port 80 for portals.

\section{Questioner: William Gropp}

Security may require more than single authentication (single point of failure). Would your users accept 2 logins or more? At what point do they throw up their hands? Follow-up: A service model that does not require accounts may provide an alternative solution. Would this be a feasible approach?

\section{David Schissel}

We always ask the question: what do users gain for what they give up. So the answer to your question, would they accept 2 logins or more, really depends on what they gain. If they perceive the gain to be small they would not be accepting of multiple logins. Our goal in FusionGrid is a onetime login for all of our services.

\section{Questioner: Dennis Gannon}

Can DOE learn from TeraGrid on the security issue?

\section{David Schissel}

The FusionGrid project has continued to reach out to other projects and other grids to learn and to make progress. Our adoption of MyProxy in the middle of our project I think is a good example of this. So the short answer to your question is yes. The longer answer is that the security requirements dictated by different government bodies (e.g. NSF vs. DOE) can change solutions from one grid to another and so the applicability of particular solutions may vary. 